\title{
Norois
}

Environnement, aménagement, société

\section{L'isolement géographique : contrainte ou opportunité pour les territoires? Regards croisés en Patagonie chilienne}

Geographic isolation: stress or opportunity for territories? Cross-perspectives through chilean Patagonia

Ingrid Peuziat, Louis Brigand, Federico Arenas, Andres Núñez et Alejandro Salazar

\section{OpenEdition}

Journals

Édition électronique

URL : http://journals.openedition.org/norois/4336

DOI : $10.4000 /$ norois. 4336

ISBN : 978-2-7535-2634-1

ISSN : $1760-8546$

Éditeur

Presses universitaires de Rennes

Édition imprimée

Date de publication : 30 décembre 2012

Pagination : 47-59

ISBN : 978-2-7535-2633-4

ISSN : 0029-182X

Référence électronique

Ingrid Peuziat, Louis Brigand, Federico Arenas, Andres Núñez et Alejandro Salazar, «L'isolement géographique : contrainte ou opportunité pour les territoires ? Regards croisés en Patagonie chilienne », Norois [En ligne], 225 | 2012, mis en ligne le 30 décembre 2014, consulté le 10 décembre 2020. URL : http://journals.openedition.org/norois/4336 ; DOI : https://doi.org/10.4000/norois.4336 


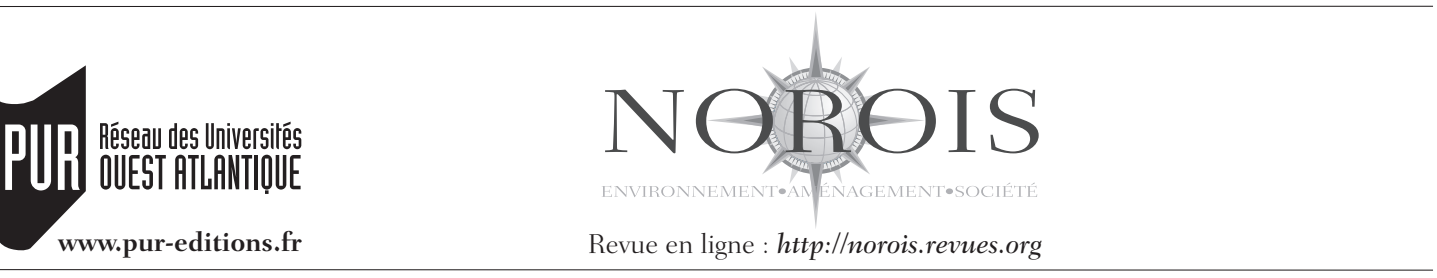

\title{
L'isolement géographique : contrainte ou opportunité pour les territoires? Regards croisés en Patagonie chilienne
}

\author{
Geographic Isolation: Stress or Opportunity for Territories? \\ Cross-perspectives through Chilean Patagonia
}

\author{
Ingrid Peuziat ${ }^{* a}$, Louis Brigand ${ }^{a}$, Federico Arenas ${ }^{b}$, Andres Núñez \\ Alejandro SALAZAR ${ }^{b}$, Hernan Escobar ${ }^{a}$
}

* auteur correspondant : ingrid.peuziat@univ-brest.fr
a LETG Brest Geomer UMR 6554 CNRS (Université de Bretagne Occidentale), Institut Universitaire Européen de
la Mer-Technopôle Brest-Iroise, place Nicolas-Copernic-29285 PlouzANÉ, France (louis.brigand@univ-brest.fr)
bInstituto de Geografia, (Pontifica Universidad Católica de Chile),Av. Vicuña Mackenna 4860, MacuL-SANTIAGo,
Chili (farenasv@uc.cl,anunez@uc.cl) (asalazar@uc.cl)

Résumé : Au Chili, la XI région (Aysen del General Carlos Ibañez del Campo) est un espace relativement isolé géographiquement. Les moyens de communication traditionnels (routes, aéroports...) restent peu développés et sont en cours de structuration. Les outils modernes (téléphonie, internet...) restent à l'état embryonnaire. Dans ce contexte, l'isolement est vécu comme une contrainte majeure dont les effets affectent tous les champs de la réalité économique, sociale et environnementale. Le peuplement actuel se concentre essentiellement dans quelques pôles urbains qui regroupent 80 \% de la population. Les $20 \%$ restants se trouvent dans des zones encore plus reculées, vallées de haute montage et îles. L'isolement dans ces sites y est encore plus manifeste. Les habitants qui y vivent développent des activités liées essentiellement à la pêche et à l'élevage extensif. Se posent pour ces populations des questions vitales de maintien de leurs activités et la prise en compte de leurs spécificités qui passent certainement par une reconnaissance régionale et des moyens nouveaux de communications. L'objet de la recherche est d'étudier la question de l'isolement dans les secteurs géographiques les plus reculés (fjords, îles et vallées montagnardes) à travers l'analyse des perceptions des populations isolées et des acteurs publics et politiques de l'Aysen. Il s'agit notamment de mettre en parallèle les atouts et les contraintes générées par l'isolement, et de conclure sur les enjeux et les perspectives qui se dressent pour ces espaces périphériques et leurs populations.

Abstract: The $11^{\text {th }}$ region (Aysen del General Carlos Ibañez del Campo) is in Chile a space in a relative geographical isolation : the traditional communications (roads, airports) are little developed and in structuralization. The modern communication tools (telephone, internet) are still in the embryonic state. In this context, the isolation is lived as a major constraint the effects of which affect all the fields of the economic, social and environmental reality. The current population is concentrated essentially in some urban poles which gather $80 \%$ of the population. The remaining $20 \%$ live in even more remote areas, mountain valleys and islands. The isolation in these sites is even more obvious. The inhabitants develop, there, activities connected essentially to fishing and to extensive breeding. At stakes for these populations are the vital questions of the preservation of their activities and the recognition of their specificities, which certainly depend on a regional recognition and new means of communications. The subject of this research is to study the question of the isolation in the most geographically remote sectors (fjords, islands and mountain valleys) through the analysis of the perception of these remote population and public and political actors in Aysen. The study analyzes the assets and constraints associated with remoteness, and concludes on the stakes and perspectives of these peripheral areas and their population. 


\begin{abstract}
Mots clés : Patagonie - Chili - isolement - développement rural - politiques publiques - perception isolement - carretera austral
Keywords : Patagonia - Chile - remoteness - rural development-public policy-remoteness perception-carretera austral
\end{abstract}

\section{INTRODUCTION : L'ISOLEMENT, UNE QUESTION GÉOGRAPHIQUE}

Bien que la notion d'isolement soit davantage abordée dans la littérature scientifique en psychologie (isolement social), en médecine, en biologie (isolement cellulaire, isolement des patients) ou en physique (isolement thermique) qu'en géographie, l'ancrage géographique de ce concept est essentiel. Dès 1653 le terme «isoler » est défini dans les dictionnaires comme suit : «faire prendre la forme d'une île ». Le mot isolement provient du latin insulatus, qui signifie changé en île, isolé, délaissé, et de insula, qui désigne une île (Gaffiot, 2001). L'isolement est donc le plus souvent appréhendé par les géographes sous l'angle de l'insularité dont il est une des composantes majeures (Bonnemaison, 1991 ; Brigand, 2002; Bernardie-Tahir, 2005; Doumenge, 1985; Pelletier, 1999; Péron, 1993 ; Taglioni, 2006). Pourtant l'isolement géographique ne concerne pas uniquement les îles, il est aussi caractéristique par exemple de certaines hautes vallées de montagne (Rieutort, 2007), d'Etats ou de territoires enclavés (Debrie et Steck, 2001 ; Gauché, 2010 ; Raballand, 2005).

A l'heure où « l'ouverture est devenue l'un des paradigmes majeurs du fonctionnement de nos sociétés » (Debrie et Steck, 2001), l'isolement est souvent perçu comme une notion négative qu'il convient de corriger en développant des actions d'aménagement visant à en diminuer les effets et à désenclaver les populations.

Pourtant, au-delà de cette logique « d'hyperconnexion et d'hyper-ouverture » matérielle ou immatérielle, on peut s'interroger sur les fonctions identitaires et l'ancrage territorial de l'isolement. Les politiques relativement standardisées d'aménagement du territoire et de gestion de l'isolement répondent-elles réellement aux attentes des populations? Les populations isolées n'adoptent-elles pas des modèles de développement spécifiques et originaux? Enfin, les territoires isolés et par conséquent les modes de vie qui s’y développent sont-ils voués à disparaître au profit d'une banalisation de l'espace humanisé?

Cette réflexion est à rapprocher de recherches antérieures, au Chili, sur les fronts pionniers halieutiques en Aysen (Peuziat, 2003), mais aussi dans les îles du Ponant (Brigand, 2002), pour lesquelles la question de l'isolement a été au cœur des politiques d'aménagement des années 1970 avec notamment l'objectif de rattraper certains retards par rapport au continent. L'opportunité d'une nouvelle recherche en Patagonie chilienne est née d'un rapprochement entre l'équipe du laboratoire LETG Brest Géomer (UMR 6554 CNRS) et l'Institut de Géographie de l'Université catholique de Santiago dans le cadre d'un programme Ecos-sud portant sur la problématique de l'isolement.

À travers l'exemple de la $\mathrm{XI}^{\mathrm{e}}$ région, Aysen del General Carlos Ibañez del Campo, composante septentrionale de la Patagonie chilienne, il s'agit de poser la question de l'isolement géographique en attachant une attention toute particulière à l'échelon local et aux perceptions des habitants, un aspect souvent négligé dans le contexte du modèle économique néo-libéral chilien et d'une centralisation forte des pouvoirs.

Après un rappel des caractéristiques des territoires isolés dans la littérature et une présentation de l'évolution de l'organisation territoriale de la région Aysen, nous analyserons et croiserons les perceptions des populations isolées et des autorités publiques sur l'isolement, pour déboucher sur les enjeux et perspectives s'offrant aujourd'hui aux populations des espaces isolés du nord de la Patagonie chilienne.

\section{L'ISOLEMENT GÉOGRAPHIQUE en Patagonie chilienne}

\section{L'isolement géographique : définitions et caractéristiques des territoires isolés}

Le géographe Roger Brunet dans Les mots de la géographie (Brunet, 2005), définit l'isolement de la manière suivante : «Sentiment lié à la faiblesse des 
relations avec les autres, avec l'extérieur ». Il peut être recherché, mais plutôt par des individus que par des sociétés. Il est mal vécu par des collectivités, des villes ou des pays, qui se plaignent volontiers de leur isolement, assimilé parfois à l'enclavement. Pourtant l'enclavement est à distinguer de l'isolement dans la mesure où celui-ci fait référence au manque d'accès d'un pays à la mer. Tous les territoires enclavés ne sont donc pas isolés et vice-versa (Raballand, 2005).

La première caractéristique d'un territoire isolé reste néanmoins liée à son cadre géographique. L'isolement géographique implique une rupture, une discontinuité physique matérialisée par exemple par une étendue d'eau séparant l'île du continent, mais aussi par le caractère accidenté d'un relief ou la rudesse du climat. Ces aspects physiques sont fondamentaux dans la littérature (Brigand, 2002; Brigand et al., 2011 ; Dodds et Royle, 2003 ; Grenier, 1997 ; Salome, 2006) et introduisent un second critère récurrent, celui de l'accessibilité ou plutôt de l'inaccessibilité des territoires isolés (Chouvy, 2003). Cette caractéristique se traduit concrètement par des réseaux de transports extrêmement faibles ou défectueux et des moyens de communication peu développés.

L'inaccessibilité et l'enclavement des territoires isolés sont aussi souvent corrélés à un faible développement économique, puisque comme le souligne P.-A. Chouvy (2003) « la route est (encore) ce moyen d'accès, ce lien physique, ce support de communication et de transport qui permet de relier les lieux de consommation et ceux de la production ». Les territoires isolés se caractérisent alors par leur exclusion ou la très faible intégration de leur économie aux flux financiers et commerciaux majeurs (Raballand, 2005). En raison notamment des surcoûts liés au manque d'infrastructures, les investissements privés y sont très limités, à moins que la présence de matières premières ne leur assure une intégration aux circuits économiques mondiaux. S. C. Carrizo et S. Velut (2005) montrent ainsi qu' "en Argentine et au Chili une phase d'insertion mondiale se met en place avec l'intégration des réseaux d'hydrocarbures à partir de 1990 ». Pour J. D. Sachs (2003) les territoires isolés sont souvent de ce fait condamnés à « des marchés internes restreints et une division inefficace du travail ». Ils sont aussi confrontés à la faiblesse des ressources naturelles et au manque de main d'œuvre. Cela est particulièrement vrai pour les petites îles comme le montre F. Doumenge (1985) qui intègre entre autres «l'exigüité du territoire, les ressources naturelles limitées et la dépendance vis-à-vis des marchés étrangers » dans ses critères de définition des micro-États insulaires.

Les territoires isolés peuvent aussi voir leur isolement accentué par des facteurs politiques et géopolitiques. L'isolement peut être en effet provoqué, à l'échelle internationale, par le régime politique. Comme l'explique P.-A. Chouvy (2003), « l'Afghanistan est devenu à partir du XIX ${ }^{\mathrm{e}}$ siècle un État-tampon que des politiques restrictives de l'accès (comprendre isolationnisme) ont confiné dans un angle géographique et géopolitique [...] qui ont considérablement accru son isolement international ». Un autre exemple est celui développé par M. E. Sili (2005) qui décrit la Terre de feu (Argentine) en ces termes : "Une des caractéristiques de cet espace est son isolement : non seulement parce que c'est une île, mais aussi parce que, pour y parvenir il faut traverser le détroit de Magellan, sous autorité chilienne. » L'isolement est également accentué lorsqu'aucune politique publique ne cherche pas à désenclaver les territoires, par l'amélioration des réseaux de transport par exemple.

On notera enfin que les territoires isolés ont généralement des structures socio-démographiques spécifiques. À l'exception de certaines îles océaniques, le peuplement y est faible et dispersé, et les territoires isolés sont bien souvent des territoires de déprise démographique. L'isolement n'encourage pas les jeunes à rester (Rieutort, 2007). On comprend donc que ces territoires doivent dans un premier temps faire face à un fort exode qui se traduit par un solde migratoire négatif. Selon J. D. Sachs (2003), « dans les pays isolés, les travailleurs sont bien plus susceptibles d'émigrer que d'attirer du capital physique dans leur pays ». Ils doivent alors dans un deuxième temps, faire face à des populations vieillissantes. L'isolement peut aller jusqu'à l'endogamie et se traduit souvent par des décalages d'évolutions sociales et culturelles dans certaines hautes vallées montagnardes et dans les îles notamment (Brigand et Le Guirriec, 1985, Brigand, 1983). Il apparaît aussi dans les études de cas des territoires isolés un déficit flagrant de structures d'éducation et de santé. 
L'isolement est donc, en géographie, la description d'une situation dans laquelle un territoire et/ou une population est séparé du reste de son environnement par un ensemble de facteurs géographiques, économiques, politiques ou sociaux. Dans nos sociétés mondialisées, hyper connectées et intégrées, quelle place (voire quelle fonction) est donnée aux isolats ? Les territoires et les populations isolées génèrent-ils ou doivent-ils générer des modèles de développement spécifiques et quelles sont leurs perspectives d'avenir?

\section{Historique du PEuplement D'UN TERRITOIRE ISOLÉ : L'AYSEN}

Au regard des critères définis précédemment, la région Aysen (XI ${ }^{\mathrm{e}}$ région) au Chili apparaît comme un territoire isolé en raison de sa situation géographique, de son étendue, de ses caractéristiques morpho-climatiques, économiques et de son peuplement relativement faible par rapport aux autres régions du Chili (figure 1 - planche III). Elle s'étend sur une surface de près de $110000 \mathrm{~km}^{2}$, soit une superficie égale en France à la Bretagne, le Centre, la Haute-Normandie et les Pays-de-la-Loire confondus. Elle se situe à un peu plus de $1300 \mathrm{~km}$ au sud de Santiago et à $600 \mathrm{~km}$ de Puerto Montt, ville au-delà de laquelle l'accès à la Patagonie par voie terrestre ne s'envisage réellement que par l'Argentine. La carretera austral, la piste reliant le nord du pays au sud créée dans les années 1970, reste difficilement praticable toute l'année et nécessite l'usage de véhicules adaptés faute de route goudronnée. Troisième région la plus vaste du Chili, l'Aysen est aussi la moins densément peuplée avec 0,9 habitants au $\mathrm{km}^{2}$ en 2002. Le peuplement de cette région s'est fait tardivement. Ce n'est en effet qu'au début du $\mathrm{xx}^{\mathrm{e}}$ siècle que l'on observe un renforcement des foyers de population existants. De plus, le peuplement de la région s'est fait en faveur de certaines localités disposant d'opportunités pour l'exploitation des ressources naturelles (élevage, bois, mine, pêche) comme Puerto Aysen et Coyhaique, deux communes qui hébergent encore en 2002 près de 8 habitants sur 10 dans la région (soit 76263 habitants) (figure 2 - planche III). Cette concentration de population se traduit aussi par un fort taux d'investissement public en termes d'infrastructures (connectivité, santé, éducation...).
Le reste de la population régionale (19715 habitants), répartie sur 8 communes, est caractérisée par sa dispersion et son isolement géographique, social, économique et politique. Les contraintes morpho-climatiques liées au relief, à l'insularité, à la forte pluviométrie et au froid compliquent encore davantage les déplacements et le déroulement des activités (Escobar, 2007). Selon une étude nationale intégrant l'ensemble de ces critères, l'Aysen compte 4 communes parmi les 20 les plus isolées du pays (figure 3 - planche IV) (Subdere, 2008; Arenas et al., 1999). On retrouvera les autres communes du Chili continental en situation d'isolement fort essentiellement dans la cordillère des Andes, au nord notamment, et dans une moindre mesure dans la cordillère de la côte. Enfin, la faible part de population rurale au Chili (13\% contre $87 \%$ d'urbains lors du dernier recensement en 2002), peut aussi être un facteur de dispersion de la population dans les espaces ruraux (figure 1 - planche III). La définition des grands axes stratégiques des politiques publiques dans la région s'est opérée en relation étroite avec les modalités de peuplement. Les premières explorations de la région remontent au $\mathrm{XVI}^{\mathrm{e}}$ siècle mais celle-ci resteront concentrées sur la partie occidentale de la région jusqu'à la fin du XIX ${ }^{\mathrm{e}}$ siècle (De Agostini, 1945). En 1907 la population régionale recensée s'élevait à 436 habitants et en 1920 à 1660 habitants. Ce n'est qu'à la fin des années 1920, avec la reconnaissance du terriroire d'Aysen par l'État central et la fondation de la ville de Coyhaique (alors appelée Baquedano), que l'on observe une augmentation significative de la population qui atteindra 9711 habitants en 1930 (Martinic, 2005).

À partir de cette date l'État intervient de manière plus directe, car jusqu'alors le développement de la région était relégué aux entreprises d'élevages et aux capitaux privés. Ce changement a été très important dans l'intégration progressive de la région au territoire national. Mais il faudra attendre 1960 pour que se mettent en place les plans de développement régionaux. Durant cette période la croissance de la population régionale se poursuit $(+240 \%$ entre 1960 et 2002) malgré une densité qui reste encore inférieure à un habitant au kilomètre carré. Ce lent processus d'intégration se voit aussi dans le fait qu'au début du $\mathrm{xxI}^{\mathrm{e}}$ siècle, $28 \%$ du territoire régional n'a encore aucun statut de propriété ni pu- 
blique, ni privée. Une grande partie de ressources naturelles n'ont pas été inventoriées ou évaluées dans leurs réelles potentialités. D’une manière générale la colonisation s'est basée sur l'exploitation des élevages bovins, à laquelle s'est ajoutée celle des ressources forestières et halieutiques. Le développement régional a ainsi été largement motivé par des intérêts économiques soutendus par le modèle néo-libéral chilien et basés sur l'extraction des matières premières (Grenier, 2003).

Dans ce contexte, les différentes stratégies publiques ont visé à intégrer les territoires pour leur valorisation à travers les activités productives et plus récemment à minimiser les effets géopolitiques de l'isolement. Ce dernier aspect s'est observé principalement sous la dictature militaire dans les années 1970 avec l'ouverture du chantier de la carretera austral dont les retombées effectives pour la région n'ont pas été à la hauteur des ambitions (Grenier, 1997). La route, largement non goudronnée, qui relie Puerto Montt ( $\mathrm{X}^{\mathrm{e}}$ région) à Coyhaique, la capitale régionale de l'Aysen (XIe région), est en effet très difficilement praticable toute l'année (dégradations liées aux intempéries, ruptures de charge liées aux passages de fjords...) et deumeure peu attractive pour les transporteurs commerciaux par exemple.

À partir de 1990, les politiques publiques s'attachent davantage à la satisfaction des nécessités de base de la population, en particulier dans les domaines de la santé, l'éducation, l'habitat et à la consolidation des voies d'accès et de communication entre les divers points régionaux (Guiñez, 1997; Contreras et al., 1998). Vers la fin de la décennie, les efforts se sont concentrés sur l'appui à la diversification économique, d'où la montée de nouveaux secteurs comme le tourisme, la pêche et l'aquaculture devançant l'exclusivité productive de l'élevage extensif qui avait perduré durant des décennies.

Au début du $\mathrm{XXI}^{\mathrm{e}}$ siècle, la région Aysen a vécu un processus qui mérite d'être souligné dans la mesure où il vient relativiser l'idée que l'isolement de l'Aysen est la cause de tous les maux de la région. En effet, dans un contexte de dégradation de l'environnement à l'échelle mondiale, la qualité et l'état de conservation des paysages patagons, et tout particulièrement de la région Aysen, sont largement reconnus. Dans ce contexte le gouvernement régional valorise de plus en plus ses aires naturelles préservées et établit dans son rapport stratégique pour la région
2000-2006 que l'« Aysen aspire à être une région décentralisée et à acquérir un niveau de qualité de vie élevé, soutenu par une croissance économique forte et équitable qui se serait fondée sur la conservation de l'environnement et l'intégration du territoire » (Subdere, 2000). Cela signifie que certains secteurs géographiques qui apparaissent aujourd'hui profondément isolés et d'une grande richesse naturelle deviendraient peu à peu des centralités dans la stratégie de développement régional. Dans la réalité, nous sommes encore loin de ce constat : même si depuis les années 1960 la question de l'isolement transparaît dans les grands axes stratégiques de développement régional en Aysen, les réponses apportées jusqu'ici se cantonnent le plus souvent à la création de routes résultant d'une vision centralisée et négative de l'isolement.

À l'heure où l'on prône la gestion intégrée et participative des territoires et leur développement durable, ces concepts ont du mal à trouver écho dans le "modèle économique chilien », faute le plus souvent de prise en compte de l'échelon local (Grenier, 2003). L'objectif de cette recherche est de reconsidérer le thème de l'isolement en Aysen et d'envisager les modalités actuelles de viabilité économique et sociale. Pour cela il apparaît nécessaire de mettre en lumière et de confronter les perceptions de cet isolement entre les habitants des secteurs les plus reculés de la région et les acteurs publics et politiques.

\section{VIVRE L'ISOLEMENT}

Le thème de l'isolement géographique préoccupe de plus en plus les acteurs en Aysen et apparaît à leurs yeux comme un enjeu majeur pour le peuplement, la planification et le développement du territoire. Pourtant l'isolement ne recouvre pas la même réalité et les mêmes enjeux pour l'ensemble des acteurs : qu'est-ce que l'isolement et quels sont ses enjeux pour les pionniers de la première heure, pour les merluceros ${ }^{1}$ des années 1980 , pour les institutionnels en charge du foncier, des infrastructures et de l'aménagement ou encore pour les élus?

Les méthodologies et les outils mis en œuvre pour répondre à ces questions relèvent à la fois de la géographie, de la sociologie et par certains aspects de

1. Nom donné aux pêcheurs artisans spécialisés dans la pêche au merlu austral (Merluccius australis). 
l'ethnologie. Une place prépondérante a été accordée au recueil d'informations à différentes échelles (locale, communale, régionale et supra régionale) en se rendant dans les territoires les plus reculés, et en rencontrant différents acteurs entre 2007 et 2010 (Arenas et al., 2010).

Le choix des communes retenues pour l'étude a été opéré en fonction de trois critères principaux : la diversité des situations géographiques (littorale ou continentale), les orientations productives des populations (élevage, exploitation forestière, pêche) et le niveau d'isolement communal conjugué à la présence de foyers de peuplement dispersés sur le territoire. Pour ce dernier aspect nous nous sommes appuyés sur le classement des communes isolées à l'échelle du Chili réalisé en 1999 et réactualisé en 2008 par l'Institut de Géographie de l'Université catholique du Chili (Santiago) et le sous-sécretariat au développement régional et administratif (figure 3 - planche IV) (Subdere, 1999 et 2008). Ce classement s'applique à toutes les communes chiliennes, à l'exception de celles de la Région Métropolitaine (Santiago). Le degré d'isolement de chaque commune est évalué selon des critères physiques (climat, éloignement à la capitale régionale, accessibilité), politico-administratifs (services publics, pouvoir décisionnel et autonomie), démographiques (croissance démographique, densité et concentration de la population), socio-culturels (indice de satisfaction des besoins de première nécessité, éducation, santé, part de la population indigène) et économiques (niveau de dépendance aux fonds externes et communaux, éloignement aux marchés). À partir de ce travail notre choix s'est porté sur cinq communes représentatives en Aysen (Cisnes, Rio Ibánez, Cochrane Tortel et Villa O'Higgins). Parmi ces communes, trois (Rio Ibánez, Villa O'Higgins et Tortel) sont en situation d'isolement très fort voire critique à l'échelle nationale : elles arrivent en $12^{\mathrm{e}}, 18^{\mathrm{e}}$ et $19^{\mathrm{e}}$ position de ce classement sur les 299 communes caractérisées au Chili pour leur isolement.

Pour le recueil des informations complémentaires à l'analyse de l'isolement des populations, les méthodologies retenues sont l'observation participante et la conduite d'entretiens semi-directifs. Ces approches ont été peu développées jusqu’alors sur ces territoires à fortes contraintes. Pour se rendre chez un éleveur de l'Avellano (Rio Ibánez) par exemple, les équipes de terrain (constituées de 2 à 5 personnes) doivent d'abord se rendre à Puerto Ibánez à plusieurs heures de pistes au sud de Coyhaique, puis entreprendre une randonnée d'une semaine à cheval dans la cordillère des Andes et parfois même traverser des fleuves en crue, pour arriver dans une exploitation de plusieurs centaines d'hectares où vit une seule famille. La rudesse du climat en Aysen nous a aussi amené à abandonner pour l'instant nos recherches sur un des terrains initialement retenus, à savoir les foyers de peuplement isolés de la commune de Villa O'Higgins, à l'extrême sud de la région. Les hivers tardifs de 2008 et 2009 n'ont pas permis à l'équipe de recherche d'envisager la chevauchée de plusieurs jours et le passage des cols encore enneigés nécessaires pour rejoindre certains foyers de peuplement. Ainsi la possibilité matérielle pour l'équipe de recherche de se rendre sur les territoires isolés est un quatrième critère qui s'ajoute au trois précédents dans le choix des secteurs étudiés. Les résultats proposés ici s'inscrivent dans une recherche exploratoire et s'appuient sur des informations recueillies au cours de cinq mois (non consécutifs) passés sur le terrain et 49 entretiens menés auprès des acteurs de l'Aysen. Ces chiffres illustrent d'une part la complexité du terrain et le temps nécessaire pour rejoindre les secteurs isolés, et d'autre part la démarche adoptée privilégiant des entrevues relativement longues (plusieurs jours) auprès de chaque famille isolée pour réaliser les entretiens (Brigand, 2009).

\section{L'isolement parfois extrême des éleveurs et des communautés de pêcheurs}

Le profil des populations vivant dans les secteurs isolés de l'Aysen varie en fonction de l'ancienneté de l'installation et des motivations de celle-ci. On distingue deux grandes catégories relatives à l'activité professionnelle : les éleveurs et les pêcheurs.

- La première concerne les populations des secteurs isolés des communes de Tortel, Cochrane et Rio Ibanez. Il s'agit le plus souvent de la première génération de pionniers et plus rarement de leurs enfants. Nés en Aysen, ils sont arrivés dans le campo à la fin des années 1970, après plusieurs semaines ou mois de voyage à travers la forêt froide tempérée à la recherche de terroirs propices aux

2. Le campo est le terme utilisé pour désigner le site sur lequel le pionner s'est installé. On y retrouve les bâtiments d'habitation ou de travail. 
activités d'élevage. Lassés des « petits boulots» d'ouvriers agricoles dans la région, ils voient dans leur installation sur des terres inoccupées, une opportunité pour construire leur vie familiale et professionnelle. Au terme de plusieurs années, ils deviennent propriétaires des terres, le Ministère des biens nationaux régularisant leur installation par l'octroi de statut de propriété foncière sur des terrains fiscaux. Aujourd'hui ces populations sont vieillissantes (plus de 50 ans) et le renouvellement des populations semble problématique. Quant aux plus jeunes (moins de 40 ans) il s'agit d'enfants nés dans le campo et qui ont fait le choix d'y rester. De moins en moins nombreux à faire ces choix, ils se retrouvent dans des situations de double isolement, à la fois géographique et humain. Le départ du campo des frères et sœurs et des voisins se conjugue souvent avec le statut de célibataire. Ces populations vivent en situation d'isolement souvent extrême : les liaisons terrestres sont difficiles et se traduisent par des déplacements à cheval de plusieurs heures, voire de plusieurs journées. Celles vivant au bord des fjords sont relativement moins isolées du fait de liaisons maritimes "régulières » (tous les 15 jours dans le meilleur des cas). Enfin, le voisinage de proximité reste très limité, la plupart des campos étant localisés à plusieurs heures de cheval ou de bateaux les uns des autres.

- La seconde catégorie concerne les populations des secteurs insulaires de la commune de Cisnes (Puerto Gala et Puerto Gaviota). Arrivées en Aysen dans les années 1980, elles sont originaires pour la plupart de la région des Lacs $\left(\mathrm{X}^{\mathrm{e}}\right)$ et des Fleuves $\left(\mathrm{XIV}^{\mathrm{e}}\right)$. Leur implantation dans les secteurs isolés est liée au développement du secteur artisanal de la pêche démersale australe en Aysen (Peuziat, 2003) et au caractère très lucratif de cette activité jusque dans le milieu des années 1990. L'isolement physique et social de ces populations est fort mais pas extrême car elles vivent en communautés d'une centaine de personnes et sont aujourd'hui reliées au chef lieu communal (Puerto Cisnes) par des liaisons maritimes une à deux fois par semaine.

\section{L'isolement au quotidien}

Qu'elles soient «pêcheurs » ou «éleveurs », les populations isolées revendiquent un mode de vie spécifique avant tout caractérisé par l'originalité de leur situation géographique à l'écart des flux et des regroupements humains. Ce choix, imposé par la recherche de sites permettant à la fois le développement d'activités primaires et la construction d'un lieu de vie, est certainement plus subi que réellement choisi. Au moment de leur installation dans les secteurs isolés, ces populations ne possèdent que peu de choses et leur faible niveau de qualification les amenaient à enchaîner les « petits boulots » d'ouvriers agricoles ou de matelot. C'est donc la contrainte économique et un certain goût de l'individualisme, doublé d'un intérêt pour la nature, qui sont à l'origine de ces installations très souvent définitives. Le pionnier, dans cette vie au cœur de la nature et des éléments, éprouve de nombreuses satisfactions. La première, c'est de construire et de réussir un projet professionnel et familial. La seconde c'est d'y trouver la liberté, de vivre dans une relative tranquillité et simplicité. S'ils apprécient globalement leur mode de vie proche de la nature, ils reconnaissent volontiers que les difficultés imposées par leur situation géographique sont réelles, notamment celles liées aux déplacements et aux conditions météorologiques. A Puerto Gala et Puerto Gaviota, la mer et le vent rendent la pêche difficile voire impossible et retardent les liaisons maritimes (figure 4). Les éleveurs du Ventisquero Montt, du Steffen ou encore du Rio Nef, sont limités dans leurs déplacements par les crues des fleuves, ou encore les glaces prises dans les fjords qui les bloquent parfois pendant plusieurs jours consécutifs (figure 5). La traversée des fleuves à cheval ou l'accès aux secteurs isolés par voie maritime sont souvent rendus délicates voire impossibles dans certaines conditions, notamment lors du relèvement des niveaux d'eau. Ces contraintes naturelles limitent considérablement les déplacements, en hiver notamment, pour les éleveurs qui sont restés au campo. Pourtant elles sont acceptées étant considérées comme un élément constitutif de leur situation géographique comme le souligne José, éleveur dans le secteur du Rio Nef (Cochrane) : «le mauvais temps, la neige et les crues du fleuve sont des contraintes majeures, parfois on ne peut pas sortir du tout d'ici, c'est l'isolement total. Pourtant c'est agréable et plus facile de vivre dans le campo que dans une grande ville ». Les difficultés de communication, liées aux transports terrestres ou maritimes, ne sont que très partiellement comblées par les télé- 


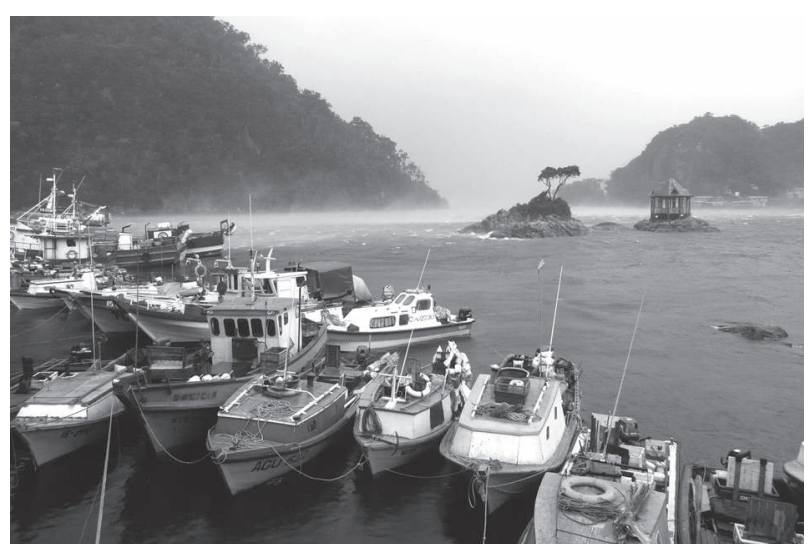

Figure 4 : botes de pêche artisanale bloqués par la tempête dans la baie de Puerto Gala (Cines).

Artisanal fisher boats (botes) stuck in Puerto Gala bay during a storm (Cisnes).

communications qui restent très limitées. À Puerto Gala et Puerto Gaviota certains commerces proposent des services téléphoniques et à Gala, le collège bénéficie même d'une connexion internet. Dans les campos isolés des communes de Cochrane, Tortel et Rio Ibanez, la radio reste le seul moyen de communiquer avec les chefs lieux communaux : trois vacations radio par jour sont généralement effectuées. Dans certains secteurs, comme la vallée de l'Avellano, un seul éleveur sur les dix présents dans ce secteur possède une radio.

Quitter le campo, quelque soit le niveau d'isolement et l'état de la météo, exige une organisation importante et reste une "expédition » de plusieurs heures, voire de plusieurs jours. Les pêcheurs attendront le bateau de liaison parfois de longues heures dans la nuit avant d'embarquer dans un petit «bote » qui les conduira à bord du «ferry » stoppé dans la baie. Atteindre le chef lieu communal demandera au mieux entre trois et cinq heures de navigation. Dans les autres secteurs littoraux que sont le Steffen, le Bajo Pascua, le Ventisquero Montt (commune de Tortel), un bateau subventionné assure la liaison avec le chef lieu communal tous les quinze jours, ce qui signifie que tout déplacement implique une absence de quinze jours hors du campo. Dans les secteurs intérieurs du Nef, du Maiten ou de l'Avellano, les éleveurs ne disposent que de leurs propres chevaux pour quitter le campo.

Le principal motif des déplacements vers les chefs lieux communaux ou de province est l'accès

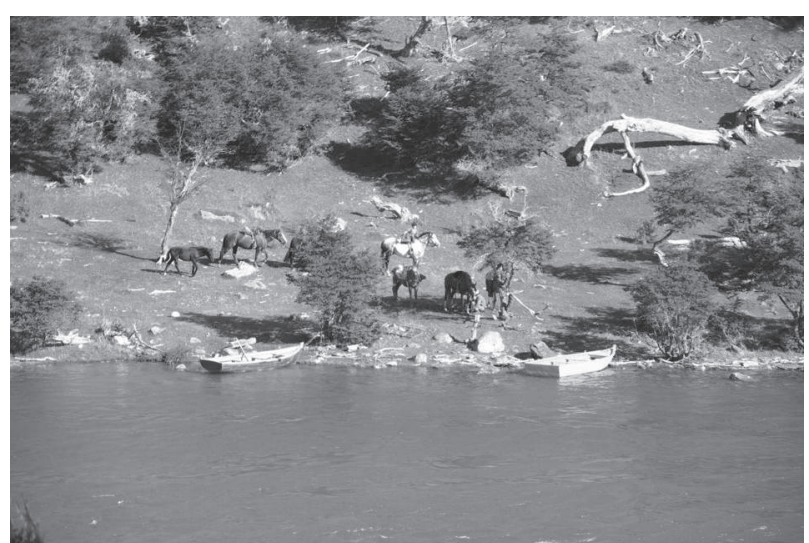

Figure 5 : L'accès au secteur du rio Nef se fait uniquement par beau temps, en barque puis à cheval. horse.

The only access to rio Nef sector is through rowing boat and

aux soins. Ainsi la fréquence des déplacements varie beaucoup en fonction de l'âge des populations. Les personnes de plus de 50 ans, souvent confrontées à des problèmes de diabète, et les jeunes mamans sont les populations qui se rendent le plus souvent chez le médecin. Elles effectuent des déplacements quasi mensuels vers les chefs lieux. Le règlement de procédures administratives relative à la pêche, au foncier ou à l'état civil ou encore les opérations bancaires sont aussi des motifs de déplacement et l'occasion de rendre visite à de la famille.

\section{La perception de l'isolement par les habitants}

Si l'isolement géographique des secteurs étudiés est important voire extrême, le sentiment d'isolement des populations ne l'est pas. En effet une large majorité des habitants disent se sentir pas trop ou pas du tout isolés. C'est le cas notamment de tous les éleveurs dont la propriété n'est desservie par aucune route et qui ne disposent que d'une liaison radio pour communiquer avec le chef lieu communal.

Il apparaît nettement que le sentiment d'isolement ou non des populations n'est pas proportionnel au niveau de communication et de télécommunication. D'ailleurs les deux enquêtés ayant manifesté un sentiment d'isolement fort sont des personnes qui souffrent davantage d'isolement social et médical, que d'isolement géographique, lorsqu'ils sont dans le campo ou sur les îles. Il s'agit aussi de personnes 
qui disposent d'une maison en milieu urbain et qui peuvent donc comparer deux modes de vie.

Malgré des conditions de vie difficiles et précaires (figures 6 et 7), les habitants des secteurs isolés ont des difficultés à évoquer les problèmes qu'ils associent à l'isolement dans leur vie au quotidien, d'abord parce qu'ils ne se sentent pas vraiment isolés, et ensuite par ce qu'ils ne perçoivent pas spontanément cet isolement de manière négative. Le témoignage d'Olga (70 ans), éleveuse dans le Steffen, est particulièrement éloquent « je suis arrivée ici à l'âge de 15 ans, juste après mon mariage, j’ai mis au monde seule onze de mes douze enfants et trois d'entreeux ont péri dans le campo avant d'atteindre l'âge de 15 ans. La vie est rude ici mais je suis heureuse, je ne souffre pas de cet isolement, c'est la vie dans le campo ». Néanmoins, après quelques instants de réflexion, le manque de services de santé est quand même le premier problème attribué à l'isolement lors des entretiens. Le vieillissement des pionniers et la forte proportion de personnes atteintes de diabète ou de maladie cardio-vasculaires rendent les populations isolées particulièrement vulnérables en termes de santé. Elles sont contraintes à des consultations régulières chez le médecin qui sont compliquées à mettre en œuvre du fait des difficultés de déplacement et d'une défaillance des services médicaux. Un pionnier vivant dans un campo de Tortel par exemple devra quitter sa propriété durant 15 jours (fréquence des rotations maritimes) pour aller prendre sa tension lors du passage de la ronde

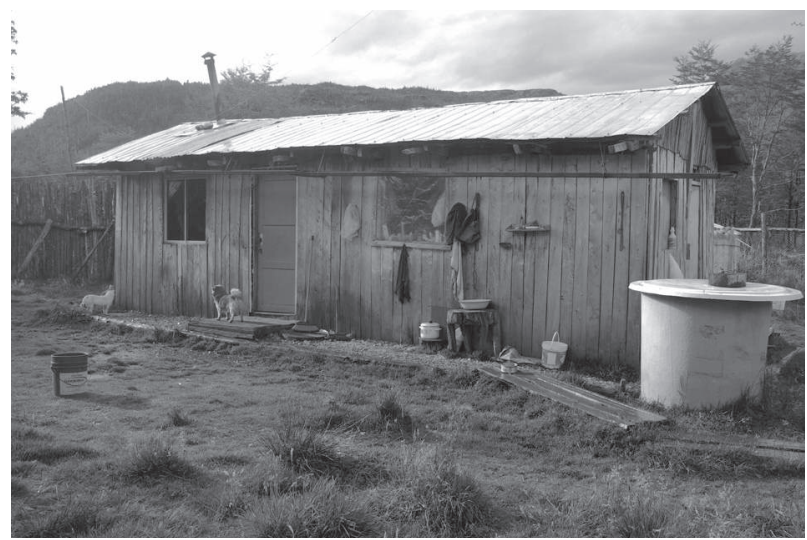

Figure 6 : Maison d'Olga, éleveuse installée depuis 55 ans dans le fjord Steffen. years ago. médicale dans le chef lieu communal. À Puerto Gala et Puerto Gaviota, les communautés disposent d'un poste de santé pour accueillir une ronde médicale normalement mensuelle mais qui dans la réalité ne passe que de manière aléatoire tous les 3 ou 4 mois environ. Les problèmes liés au manque d'ouverture sur l'extérieur, à savoir le faible taux d'équipement en réseau de communications ou encore le sentiment d'isolement social fort, sont aussi cités comme des difficultés de la vie dans l'isolement mais arrivent très loin derrière les préoccupations de santé. Les pionniers ou leurs descendants vivant dans les campos de plusieurs centaines d'hectares ont développé des modes de vie spécifiques dont les exigences temporelles sont essentiellement celles imposées par la nature (les saisons, la météorologie, l'entretien du bétail et d'un petit potager...) et dont les besoins sont souvent réduits au strict nécessaire pour le bien être de la famille et des animaux. À Puerto Gala et Puerto Gaviota, malgré une ouverture plus grande liée au contexte socioéconomique de la pêche (migration de travailleurs, achat des produits de la pêche par des entreprises extérieures...), l'isolement reste à leurs yeux une valeur essentielle dans les modes de vie et pour la pérennité des communautés insulaires.

Aussi pour tous, éleveurs ou pêcheurs, l'isolement semble être le garant qu'une qualité de vie perdue dans les «villes » et caractérisée par la tranquillité et la liberté.

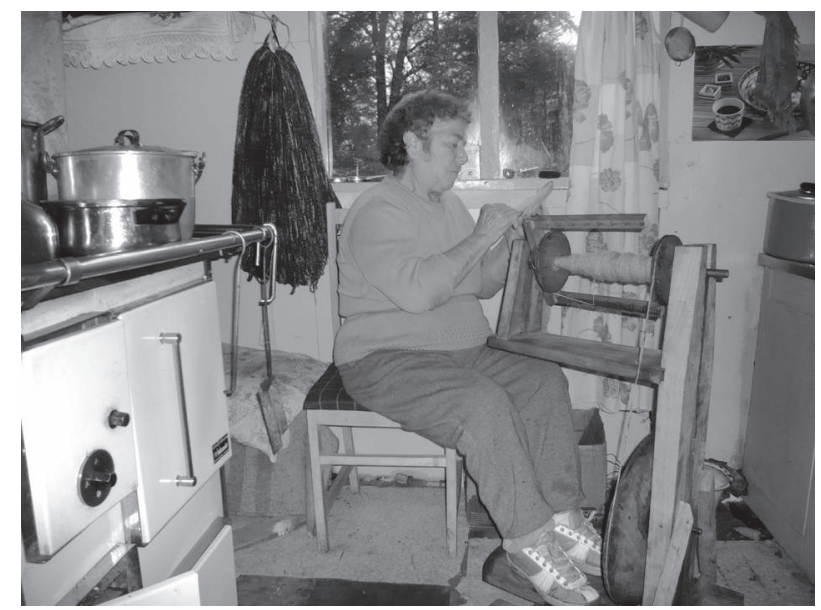

Figure 7 : Olga tissant la laine de ses moutons devant la « cosinera de lena ", l'unique moyen de chauffage et de cuisson.

Olga weaving her sheep wool in front of the wood stove, the only mean of heating And cooking in the house. 


\section{GÉRER L'ISOLEMENT Quel isolement?}

Au-delà du recueil des points de vue des populations isolées, l'originalité de ce travail réside dans leur confrontation avec ceux des élus locaux (maires notamment) et des administrations régionales ayant des compétences dans la gestion des territoires isolés en relation avec le foncier, les réseaux de communication et de télécommunication ou encore les espaces forestiers et/ou protégés.

Il est d'abord intéressant de noter qu'il n'existe pas une définition unanime de l'isolement. Là où les populations isolées ont des difficultés à donner des critères pour définir l'isolement car elles ne se sentent pas fondamentalement isolées, les élus diront que l'éloignement aux marchés et le manque de route sont les éléments qui caractérisent les territoires isolés. Parmi les administrations deux tendances de dégagent :

- Les premiers attribuent l'isolement à un déficit en terme de communication (routes, télécommunications...) et de services (écoles, médecins, poste, animation culturelles...). Il s'agit des organismes en charge du foncier, des espaces protégés et de l'aménagement (Bienes Nacionales, Sistema Nacional de Áreas Silvestres Protegidas del Estado et Ministerio de Planificacion).

- Les seconds définissent l'isolement comme une notion extrêmement subjective en relation avec le sentiment d'isolement de la part d'une population et non directement avec le manque de connectivité. Ce qui implique qu'un territoire ne peut être isolé que s'il est occupé. Il s'agit des organismes en charge des transports, de la construction de routes (Ministerio de Obras Públicas, Seremi transporte Telecomunicación) et des forêts (Corporación Nacional Forestal-Conaf).

L'isolement est perçu comme un problème majeur par les élus et non par les administrations ou encore les populations isolées comme nous l'avons vu plus haut. L'isolement constitue pour eux un surcoût pour la population et les acteurs économiques. Il freine le développement économique, démographique et culturel de leur commune. Le maire de la commune de Cochrane est l'élu qui se sent le plus isolé alors qu'en terme de connectivité et d'équipement (collège, hôpital...), par exemple, il ne s'agit pas de la commune la plus isolée.
Pour l'ensemble des administrations, l'isolement de certains secteurs de l'Aysen est plutôt un atout qui octroie une valeur intrinsèque au territoire, "valeur qui devrait croître de manière exponentielle avec le temps, dans nos sociétés hyper connectées où il devient difficile de faire l'expérience de l'isolement » selon la Conaf (Corporation nationale forestière).

\section{L'adéquation des politiques publiques et des attentes des populations isolées}

Considérant la diversité des points de vue sur l'isolement on peut s'interroger sur l'adéquation entre les actions menées ou envisagées par les organismes publics en relation avec les problématiques d'isolement et les attentes réelles des populations isolées. L'un des premiers résultats marquants de cette analyse est le fait que les populations des secteurs isolés disent souffrir «d'isolement politique ». Un sentiment davantage lié au manque de considération et de reconnaissance de leurs spécificités par l'État et les élus, qu'au manque d'actions concrètes en faveur de la connectivité, de l'amélioration de l'habitat ou encore de l'octroi de subventions.

Dans ce contexte on notera que de prime abord les populations isolées disent avoir peu d'attentes vis-à-vis de l'État, non par faute de besoins mais plutôt par résignation. Certains diront «ne pas attendre grand-chose des autorités car ils estiment ne pas être considérés ». D'autres, ayant conservé l'état d'esprit des pionniers, n'attendent d'une manière générale rien de personne et estiment devoir se débrouiller seuls.

Néanmoins le discours n'est pas totalement sans contradictions car au-delà de ces premières considérations, ils aimeraient quand même une intervention publique dans les secteurs suivants:

- L'amélioration des moyens de communication : construction de routes, chemins, passerelles, amélioration de la fréquence et de la sécurité des transports maritimes subventionnés.

- Pour les pêcheurs, le soutien aux activités halieutiques et à la diversification économique : aides à la pêche, création d'un statut pour la pêche artisanale comme une activité durable par rapport à la pêche industrielle et la salmoniculture.

- L'amélioration de l'accès aux soins : rondes médicales à améliorer ou à créer. 
- L'amélioration de l'habitat et des bâtiments d'élevage.

Les entretiens avec les organismes publics et les élus montrent que d'une manière générale les actions en cours et envisagées répondent en apparence aux attentes des populations :

- Soutenir les projets touristiques.

- Encourager la gestion participative des espaces forestiers par les populations locales.

- Construire des routes.

- Améliorer la qualité, la fréquence et la sécurité dans les transports pour les populations locales et les touristes.

- Lutter contre l'isolement technologique (télécommunication).

- Améliorer prioritairement les réseaux d'eau potable, d'assainissement et d'électricité et dans un second temps les services de santé, et d'éducation.

Dans le détail on notera pourtant certaines limites. L'amélioration des services de santé dans les secteurs isolés par exemple n'apparaît pas comme une priorité alors que le problème d'accès aux soins est une cause importante d'exode des populations isolées vers des chefs lieux communaux ou régionaux équipés d'un hôpital. Le manque de soutien à la diversification économique est aussi notable. Pourtant le maintien des activités, voire l'encouragement à la pluriactivité, sera probablement essentiel au maintien des populations dans les secteurs isolés. Le soutien aux projets touristiques évoqués par les administrations pose la question des capacités économiques et de la formation des populations isolées de l'Aysen pour entreprendre ces projets localement. Plusieurs personnes rencontrées au cours des entretiens ont mentionné des tentatives de mise en place d'activités touristiques, comme par exemple la randonnée équestre, mais tous ces essais se sont soldés par des échecs. Pourtant, les plus jeunes évoquent régulièrement l'idée que l'une des solutions les plus adaptées à leur survie serait un tourisme de nature axé sur des activités de découverte des grands espaces. On peut cependant s'interroger légitimement sur la faisabilité et la rentabilité d'activités touristiques sur ces secteurs, en raison des coûts d'acheminement et de la qualité actuelle des hébergements qui hypothèquent tout espoir d'un développement touristique d'une certaine ampleur. En revanche, la mise en place par des étrangers de lodges semblent une hypothèse envisageable. On peut craindre que le développement touristique ne se fasse uniquement au profit de grands opérateurs touristiques ou des multinationales, à l'image des cycles économiques passés de la région?

\section{Conclusion : UN AVENIR INCERTAIN}

Lorsque l'on aborde la question de l'avenir des territoires auprès des populations isolées, plusieurs problématiques sont évoquées. Elles se rattachent essentiellement à trois questions cruciales, à savoir le déclin démographique des campos, le statut de la propriété foncière et les choix en matière économique.

\section{Le déclin démographique}

La situation démographique des territoires isolés est préoccupante. À Puerto Gala et Puerto Gala la population stagne, les effectifs des écoles déclinent tandis que dans les campos des communes de Cochrane, Tortel et de Rio Ibanez la population vieillit et les perspectives de reprise des propriétés par les héritiers n'est pas toujours assurée.

\section{La problématique foncière}

Cette question préoccupe particulièrement des populations d'éleveurs des communes de Cochrane et de Tortel. La pression foncière se renforce de manière importante dans ces secteurs, notamment dans le contexte du développement de projets écotouristiques à proximité des grands glaciers continentaux, les Campos de hielo Sur et Norte, et des projets de création de barrages hydro-électriques sur les fleuves Baker et Pascua, par le groupe Endesa.

Dans ce contexte les éleveurs en cours de régularisation sur des terrains fiscaux craignent que le ministère des biens nationaux ne leur octroie plus de titre de propriété, pour pouvoir vendre à des promoteurs ou limiter les transferts fonciers. Les pionniers de la première heure s'interrogent sur leur succession et sur la possibilité de vendre. Les plus jeunes éleveurs savent qu'ils devront diversifier leur activité professionnelle, probablement en développant des petits projets touristiques mais là encore ils craignent de ne pas obtenir de servitude de passage sur les terres situées entre leur propriété et les grands sites attractifs de la région (fleuves pour la pêche sportive, glaciers...). 
La propriété foncière est une question essentielle pour le développement des territoires et des populations en Aysen comme ailleurs et sur ce plan les populations isolées des communes étudiées semblent être dans une période charnière.

\section{La diversification économique dans les secteurs isolés}

Si la question de la diversification économique se pose dans une certaine mesure pour les éleveurs comme nous l'avons vu plus haut, elle est cruciale pour les pêcheurs de Puerto Gala et de Puerto Gaviota. Tous les hommes et femmes enquêtés dans ces secteurs associent l'avenir des villages et de leurs habitants à celui de la pêche artisanale en Aysen. La modification des réglementations et l'instauration des quotas individuels a largement fragilisé le processus de sédentarisation des pêcheurs dans ces secteurs insulaires. La rentabilité économique de l'activité est moindre et pour certains si cette tendance perdure sans que soit développés d'autres pêcheries, de nouveaux circuits de transformation ou de commercialisation du poisson voire d'autres activités économiques, l'avenir des communautés sera très difficile. Les pêcheurs ne sont pas optimistes et cela d'autant plus que les villages s'ouvrent de plus en plus sur l'extérieur et que la cohésion communautaire fait de plus en plus défaut dans un contexte d'incertitudes.

Pour les administrations enquêtées, l'avenir des territoires isolés et de leurs habitants est souvent associé à la valorisation des potentialités touristiques de la nature de l'Aysen et de l'isolement. La régularisation foncière est aussi un élément essentiel pour permettre le développement touristique. Les plus optimistes espèrent que l'isolement génèrera des modèles de développement spécifiques encore à inventer.

En conclusion, on peut s'interroger sur le devenir de ces populations isolées. Si les habitants des campos restent très attachés à leur territoire et à leurs propriétés, les évolutions constatées notamment au plan démographique sont inquiétantes. Le maintien des familles n'est plus assuré et certains campos deviennent progressivement des résidences secondaires. La revendication d'un mode de vie spécifique, fondé sur l'usage du bateau ou du cheval et par l'éloignement géographique au village, constitue un élément constitutif du discours des pionniers. La plupart des individus rencontrés au cours de nos enquêtes revendiquent le plaisir de vivre dans la nature qui forge une identité patagonne. Ce mode de vie qui s'appuie sur des activités primaires et sur une relation très proche avec le territoire impliquent des conditions de vie très humbles, qui ne sont pas évoquées comme un problème mais plutôt comme un certain art de vivre.

On peut raisonnablement penser que l'avenir des populations isolées reste très incertain. Si l'opposition au projet de construction de barrages hydro-électriques fait presque l'unanimité auprès des habitants et des représentants d'organismes publics enquêtés, tous s'accordent à dire que ces équipements, s'ils voient le jour, conduiront à de grands bouleversements démographiques, sociaux et économiques. La question de l'isolement sera alors totalement reconsidérée. Ainsi la situation des populations les plus isolées présentée dans le cadre de cette étude témoigne de modes de vie en voie de disparition et cela, en dépit du fait que sur de nombreux aspects, ils répondent aux critères développés à l'échelle internationale pour la définition de modes de vie durables (PNUE, 2009).

\section{Remerciements}

Les auteurs remercient le programme ECOS SudCONICYT pour sa contribution au financement de cette recherche.

\section{Bibliographie}

Arenas, F., Brigand, Escobar H., L., Núñez; A., Peuziat, I., Salazar, A., 2010. Informe Final del proyecto de intercambio de programa de cooperación científica ECOS-CONICYT (Código : C06H03), El aislamiento geográfico en Patagonia Chilena, Región de Aysén, ventajas y problemas. Géomer CNRS UMR 6554. Institut Universitaire Européenne de la Mer/Université de Bretagne Occidental, Francia/Instituto de Geografía/Pontificia Universidad Católica de Chile. 142 p.

Arenas F., Quense J., Salazar A., 1999. "El aislamiento como desafío para el ordenamiento territorial. El caso de las comunas de Chile", Revista de Geografía Norte Grande, $\mathrm{n}^{\circ} 26$, p. 105-111

Bernardie-Tahir, N., 2005, Des «bouts du monde » à quelques heures : l'illusion de l'isolement dans les petites îles touristiques, Annales de Géographie n 644, Armand Colin, p. 362-382. 
Brigand L., Peuziat, I., Arenas, F., Salazar A., Núñez, A Escobar H., 2011. Aislamiento geográfico en las islas costeras francesas y en la Región de Aysén en Patagonia chilena : primeros elementos comparativos, dans Arenas F., Salazar A., NuÑEz A., El aislamiento geográfico : problema u oportunidad? Experiencias, interpretaciones y politicas públicas. Serie Geolibros, Pontifica Universidad Catolica de Chile, p. 23-33.

Brigand L., 2009, Besoin d'îles, Stock, 252 p.

Brigand L., 2002, Les îles du Ponant. Histoires et géographie des îles et archipels de la Manche et de l'Atlantique, Plomelin, Palantines, $480 \mathrm{p}$.

Brigand L., Le Guirriec P., 1985. Insularité et choix du conjoint. Le cas de l'île de Batz à la fin du XIX et au début du $x^{e}$ siècle in Actes du Colloque 1984: "Le littoral, milieux et sociétés ", Anthropologie maritime, n² 2, p. 115 122.

Brigand L., 1983. Les îles bretonnes aspects géographiques de l'insularié, thèse de Géographie, Université de Bretagne Occidentale, $267 \mathrm{p}$.

Brunet R., 2005. Les Mots de la géographie, Paris, La documentation française, coll. "Dynamiques », 518 p.

Bonnemaison, J., 1991. Vivre dans une île, une aproche de l'îléité océanienne, L'espace géographique, p. 119-125.

Carrizo S. C., Vélut S., 2005. Nouvelles territorialités en Amérique australe. Activités énergétiques et intégration dans les terres et les mers magellanes, L'Espace géographique 2/2005 (tome 34), p. 161-175.

Chouvy P.-A., 2003. La production illicite d'opium en Afghanistan dans le contexte de l'enclavement, de l'isolement et de l'isolationnisme, Cahiers d'Études sur la Méditerranée Orientale et le monde Turco-Iranien, ${ }^{\circ}$ 35, p. 71-82.

Contreras P., Henrique Ch., Qüense J., 1998. Gestión de un instrumento de planificación territorial en una localidad aislada : Lago Verge XI Región de Aysén, Chile, Revista de Geografía Norte Grande, n² 25, p. 73-80.

De Agostini A., 1945. Andes Patagonicos, Buenos Aires, 444 p.

Debrie J., Steck B., 2001. L'enclavement, réévaluation théorique et application à l'Afrique de l'Ouest, L'Espace géographique, $\mathrm{n}^{\circ} 1 / 2001$, tome 30 , p. 26-36.

Dodds K., Royle S. A., 2003. The historical geography of islands. Introduction : rethinking islands, Journal of historical geography, vol. 29/4, p. 487-498.

Doumenge F., 1985. Les îles et les micro-États insulaires, Hérodote, n³ 37-38, p. 297-327.

Escobar H., 2007. L'organisation territoriale en Patagonie chilienne: enjeux et perspectives pour la région d'Aysen. Thèse de doctorat de Géographie, Géomer UMR 6554, Université de Bretagne Occidentale, 276 p.

GafFiot F., 2001. Dictionnaire Latin-Français, Hachette éducation, $820 \mathrm{p}$.

Gauché E., 2010. Le désenclavement des territoires ruraux marginalisés du Nord du Maroc : les conséquences de la nouvelle route côtière méditerranéenne dans le Rif oriental (territoire des Beni Saïd), Norois, n² 214, p. 67-84.

Guiñez H., 1997. La estratégia de desarrollo de la XI Región, de Aysen : Un enfoque geográfico, Revista de Geografía Norte Grande, $\mathrm{n}^{\circ} 24$, p. 59-64.

Grenier P., 2003. Des tyrannosaures dans le paradis : La ruée des transnationales sur la Patagonie chilienne, Librairie L'Atalante, $347 \mathrm{p}$.

Grenier P., 1997, L'ouverture de la « Carretera Austral » dans les Andes du Chili méridional : quel modèle de développement touristique soutenable?, Revue de géographie alpine, $\mathrm{n}^{\circ} 85-1$, p. $75-85$.

Martinió M. B., 2005. De la Trapananda al Aysen, Biblioteca del Bicentenario, Pehuen, 539 p.

Pelletier P., 1999. Iles éloignées, passages obligés : le rôle de la surinsularité dans la civilisation japonaise in L'Île Laboratoire, Anne Meistersheim éd., Ajaccio, Éditions Alain Piazzola, 464 p., p. 301-317.

PÉron F., 1993. Des îles et des hommes : l'insularité aujourd'hui, Rennes, Éd. de la Cité/Ouest-France, 286 p.

Peuziat, I., 2003. Chili: les gitans de la Mer, pêche nomade et colonisation en Patagonie chilienne, Paris, L'Harmattan, 2006 p.

Pnue, 2009. Groupe de travail sur les modes de vie durable, Suède, PNUE, $67 \mathrm{p}$.

Raballand G., 2005. L'Asie centrale ou la fatalité de l'enclavement, Paris, L'Hamattan, collection « Centre-Asie », 355 p.

Rieutort L., 2007. Du territoire identitaire aux nouveaux partenariats ville-campagne : les voies du développement local dans la haute vallée de la Loire, Norois, vol. 3/2007, n² 204, p. 11-23.

SACHS J. D., 2003. Les institutions n'expliquent pas tout. Le rôle de la géographie et des ressources naturelles dans le développement ne doit pas être sous-estimé, Finances et développement, juin 2003, Fonds Monétaire International, p. 38-41.

SALOMÉ K., 2006, Les îles bretonnes au XIX ${ }^{\mathrm{e}}$ siècle entre éloignement et isolement, Ethnologie française, XXXVI, n 3 , vol 36, p. 435-441.

Sili M. E., 2005. La Terre de Feu face à l'avenir. De la crise du territoire à la construction d'un nouveau mythe de développement, L'Espace géographique, vol. 1/2005 (tome 34), p. 17-27.

Subdere (Subsecretaría de Desarrollo Regional), 2008. Actualización estudio diagnostico y propuesta para territorios aislados, $40 \mathrm{p}$.

Subdere (Subsecretaría de Desarrollo Regional), 2000. Estrategia Regional de Desarrollo para la Región de Aysén 2000-2006, Ministerio de Planificación y Cooperación, región de Aysén, 53 p.

TAGLioni F., 2006. Les petits espaces insulaires face à la variabilité de leur insularité et de leur statut politique, Les Annales de géographie, $\mathrm{n}^{\circ}$ 652, p. 664-687. 